J. Lake Sci. (湖泊科学), $2006, \mathbf{1 8}(4): 363-368$

http:// www. jlakes. org. E-mail: jlakes@ niglas. ac.cn

(c) 2006 by Journal of Lake Sciences

\title{
沉积物金属元素变化的粒度效应——太湖沉积岩芯为例"
}

\author{
刘恩峰, 沈吉, 朱育新 \\ (中国科学院南京地理与湖泊研究所,南京 210008)
}

\begin{abstract}
摘 要: 水体沉积物中金属元素含量变化除了与人为污染有关之外, 在很大程度上受沉积物粒度、矿物组成等沉积物性质 的影响. 在进行沉积物金属元素研究中, 要充分考虑沉积物金属元素含量变化的粒度效应. 本文通过对太湖 MS 岩芯中 17 种金属元素、沉积物粒度、矿物组成等指标的分析, 研究了金属元素变化特征以及与沉积物粒度组成的关系. MS 岩芯金属 元素变化可分为两类, 第一类主要为 $\mathrm{Al} 、 \mathrm{Fe} 、 \mathrm{~K}$ 等, 该类元素在沉积岩芯中下部含量较高, 岩芯上部含量较低; 第二类为 $\mathrm{Na}$ 元素, 在沉积岩芯中下部含量较低, 岩芯上部含量升高. MS 岩芯中金属元素与粘土含量之间具有显著相关关系, 经沉积物 粒度 (粘土含量) 校正后, 沉积岩芯中金属元素含量趋于稳定. 因此, 沉积物粒度组成 (粘土含量) 是影响金属元素含量的 主要因素, 在研究太湖沉积物金属元素变化规律及进行金属污染评价时,应对金属元素含量进行粒度校正.
\end{abstract}

关键词: 沉积物; 金属元素; 粒度; 含量校正;太湖

\section{The influence of grain size composition on metal content in the sediment: the case of core sediment in Lake Taihu}

\section{LIU Enfeng, SHEN Ji \& ZHU Yuxin}

( Nanjing Institute of Geography \& Limnology, Chinese Academy of Sciences, Nanjing 210008, P. R. China )

\begin{abstract}
The metal contents in the sediments are influenced not only by human pollutants, but also by grain size and mineral composition of the sediments, which should be evaluated in the study of heavy metal. The metals contents and their correlations with grain size in sediments of core MS from Lake Taihu are studied in this paper in order to evaluate the grain size effects on metals contents. The metals in core MS can be classified into two categories according to their variation characteristics. Al, Fe, K etc belong to one category, the contents of which are high in the middle-lower section of the sediment core and low in the upper section; Na belongs to the other category, the variation of its content is opposite with the first category. According to the correlations of the metals and grain size, clay content was selected as the normalization component of metals. After normalized by the clay content, the metals contents take on consistent variation along the sediment core. The analysis results show that the metals contents in Core MS are influenced mainly by grain sizes composition and the normalization of metals content must be taken during the study of metals variation and pollution evaluation.
\end{abstract}

Keywords:Sediments; metals; grain size; normalization; Lake Taihu

近年来, 随着工农业污染物排放量的不断增加, 水环境质量日趋恶化,其中水体沉积物中具有潜在生态 毒性的金属元素污染备受广泛关注 ${ }^{[1-16]}$. 沉积物中金属元素含量除了受到人为污染而发生改变之外,粒 度、矿物组成等沉积物性质的差异, 使不同水体沉积物、甚至同一水体沉积物不同样品中的金属元素含量也 不同 ${ }^{[2-4,17-20]}$. 国内外学者对沉积物金属元素含量与粒度的关系进行了一定的研究,陈中原等对长江口表 层沉积物及岩芯中金属元素含量与粒度关系的研究、张朝生等对渤海湾表层沉积物金属元素含量与粒度关 系的研究、Roussiez 等对地中海西北部 Lions 湾沉积物金属元素与粒度等变化规律的研究都表明,沉积物金

* 中国科学院知识创新工程重要方向项目 (KZCX3 - SW - 348)、中国科学院知识创新工程重大项目 (KZCX1 - SW 12 - I ) 共同资助. 2005-08-05 收稿;2005-11-11 收修改稿. 刘恩峰,男,1976 年生,博士, 助理研究员; E-mail: efliu@ niglas. ac.cn. 
属元素含量直接受到沉积物粒度变化的影响 ${ }^{[4,19,20]}$; 粗颗粒沉积物中 $\mathrm{SiO}_{2}$ 含量较高, 从而对沉积物中其它 元素产生 “稀释作用” ${ }^{[4,17-19]}$. 因此, 在进行沉积物金属元素含量对比分析、污染评价时, 都应消除或减小沉 积物粒度、矿物组成变化对金属元素含量的影响, 对金属元素含量进行校正 ${ }^{[2-5,17-22]}$. 目前通常采用的校正 方法为沉积物粒度校正和参比元素校正. 沉积物粒度校正可分为两类, 一是直接分析沉积物细颗粒某一粒 级中金属元素的含量; 二是分析整个样品中金属元素含量, 然后校正到某一粒级金属元素含量 ${ }^{[5,20,21]}$. 参比 元素校正是选择一种或几种元素作为可对比的标准元素, 这种元素在自然条件下与目标元素 (污染元素) 含量具有相似的变化规律, 且基本不受人类活动的污染, 通常所采用的参比元素为 $\mathrm{Al} 、 \mathrm{Fe} 、 \mathrm{Cs} 、 \mathrm{Sc} 、 \mathrm{Co}$ 和 $\mathrm{Ti}$ 等 $[3,4,17,20,22]$.

有关太湖沉积物金属含量以及污染等问题开展了一定研究 ${ }^{[5-11,23]}$, 但由于太湖不同湖区沉积物粒度 组成存在差异 ${ }^{[23]}$, 同时缺乏沉积物中金属元素含量与沉积物粒度组成关系的系统研究, 使得太湖沉积物金 属污染研究成果的对比缺乏科学性. 本文拟通过沉积岩芯金属元素含量、粒度等指标的分析, 初步探讨太湖 沉积物金属元素含量与沉积物粒度变化之间的关系、以及沉积物中金属元素含量的标准化方法.

\section{1 材料与试验方法}

本文分析样品采用 2002 年 10 月在太湖马迹山附近湖区 ( N31 ${ }^{\circ} 22^{\prime} 55.7^{\prime \prime}$; E120 $\left.07^{\prime} 56.0^{\prime \prime}\right)$ 采集的长 50 $\mathrm{cm}$ 的柱状沉积岩芯 MS(图 1), 取样间隔为 $0.5 \mathrm{~cm}$, 样品量为 100 个.

用于沉积物粒度分析的样品用稀盐酸、双氧水处理 ${ }^{[24]}$, 分 别去处碳酸盐和有机质后, 用英国 Malvern 公司生产的 Mastersizer - 2000 型激光粒度仪进行分析, 样品平行分析误差小于 $5 \%$. 用于沉积物金属元素含量分析的样品采用盐酸 - 硝酸 氢氟酸消化法处理后 ${ }^{[24]}$, 用美国 LEEMAN LABS PROFILE 型 电感耦合等离子体原子发射光谱仪 (ICP - AES) 测定, 标样中 各元素的实测值误差均小于 $5 \%$.

\section{2 结果与分析}

\section{1 沉积物粒度变化特征}

太湖 MS 沉积岩芯粒度组成以粘土和细粉砂为主, 岩芯中 下部粒度组成相对较细, 而岩芯上部粒度组成明显变粗 (图 2). 根据沉积物粒度频率曲线、 $\mathrm{C}-\mathrm{M}$ 分布图、以及沉积岩芯 ${ }^{137}$ Cs 、 ${ }^{14} \mathrm{C}$ 测年分析结果, MS 岩芯为统一大太湖形成后的典型湖 相沉积物, 受扰动作用较弱 ${ }^{[24]}$. 太湖沉积物主要是来源于太湖 流域的西部及南部山区 ${ }^{[23]}$,物源区面积较大, 小流域的物源改 变对太湖沉积物来源的影响较有限, 因此历史时期沉积物母

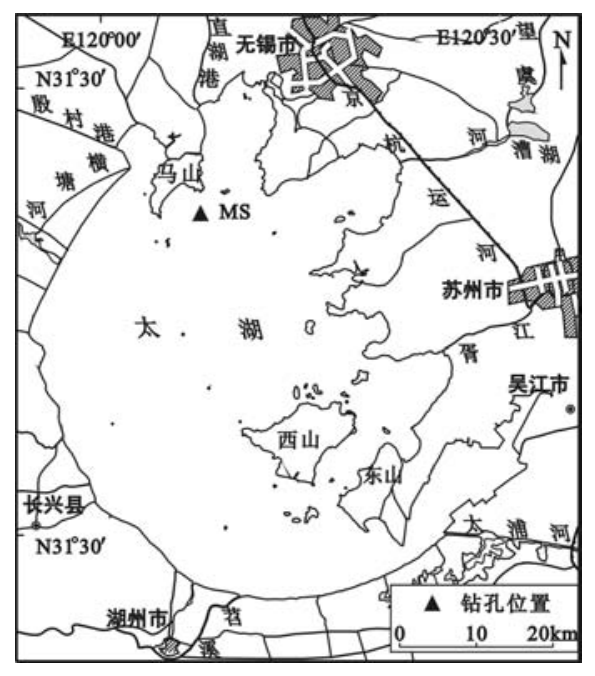

图 1 MS 岩芯位置

Fig. 1 Core site of MS in Lake Taihu 质来源应基本相似.

参照中国国家海洋局粒度粒级分类标准 ${ }^{[25]}$, 对 MS 岩芯粒度组成分成 5 个组分, 进行主成分分析. 分析 结果表明, 前两个主成分贡献率为 $82.4 \%$, 其包含的信息量已经达到统计学要求. 其中第一个主成分贡献 率为 $60.9 \%$, 主要为粘土 $(<4 \mu \mathrm{m})$ 、极细粉砂组分 $(4-8 \mu \mathrm{m})$, 以及粗颗粒组分 $(>64 \mu \mathrm{m})$; 第二主成分中 贡献率为 $21.5 \%$, 主要为 $16-64 \mu \mathrm{m}$ 组分 (图 3).

\section{2 金属元素变化特征}

MS 岩芯中金属元素与粒度具有相似的变化特征, 根据金属元素 Q 型聚类分析结果, 金属元素含量变 化可分为两类, 第一类元素包括 $\mathrm{Al} 、 \mathrm{Fe} 、 \mathrm{~K}$ 等, 第二类是 $\mathrm{Na}$ 元素. MS 沉积岩芯中下部第一类元素含量较高, $\mathrm{Na}$ 含量较低; 岩芯上部, 第一类元素含量相对较低, $\mathrm{Na}$ 含量相对较高 (图 2). 相关分析结果表明, MS 沉积岩 芯金属元素之间具有较好的相关性,而且第一类元素间呈显著正相关,第一类元素与第二类元素之间呈显 著负相关 (表 1 ). 


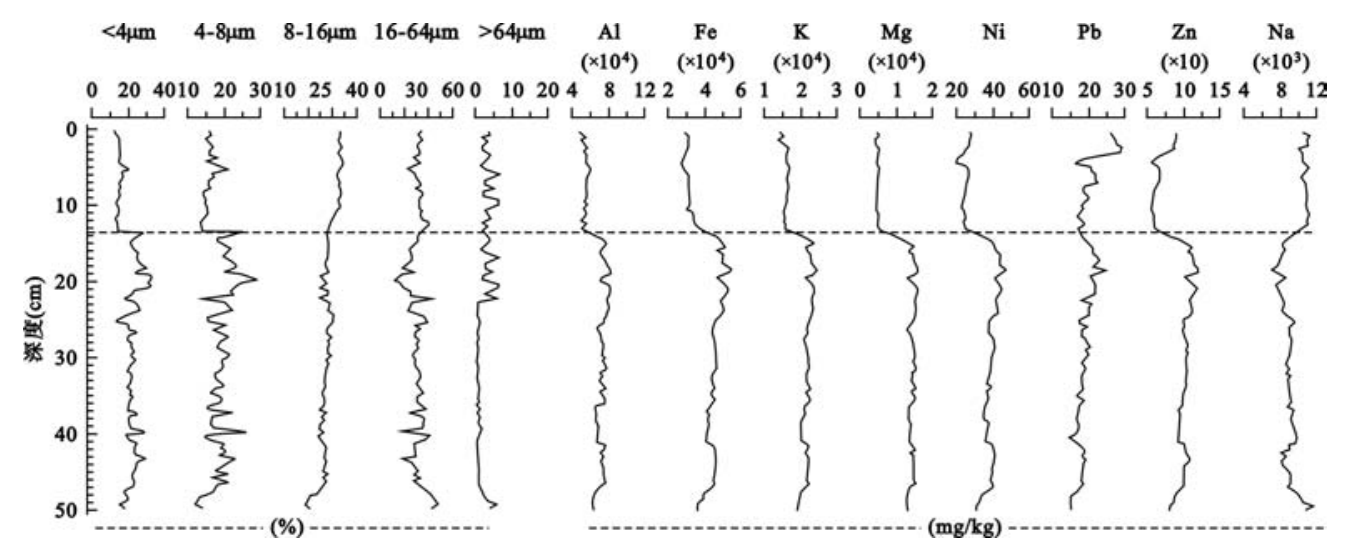

图 2 MS 岩芯沉积物粒度、部分金属元素含量变化

Fig. 2 The distribution of grain size and metals in Core MS of Lake Taihu

在水体沉积物金属元素污染评价时,对于沉积物粒度 组成变化不大的样品,通常采用未受人为污染的表层沉积 物、或者沉积岩芯底部样品中金属元素含量作为背景 值 ${ }^{[4,5,12]}$. 就 MS 岩芯金属元素变化规律而言, 如果采用岩 芯下部金属元素含量作为背景值, 沉积岩芯上部 $\mathrm{Pb} 、 \mathrm{Na}$ 等 元素已明显受到人为污染,这显然与 $\mathrm{Na}$ 为非人为污染元 素相矛盾. MS 岩芯表层沉积物金属元素形态分析结果表 明, 只有 $0-4 \mathrm{~cm}$ 沉积物中 $\mathrm{Cu} 、 \mathrm{Mn} 、 \mathrm{Ni} 、 \mathrm{~Pb} 、 \mathrm{Zn}$ 受到一定程 度的人为污染 ${ }^{[7]}$. 因此, 采用沉积岩芯底部金属元素含量 作为背景值进行元素污染评价显然不妥.

\section{3 金属元素与粒度变化的关系}

不同粒级沉积物由于其矿物组成、结构和表面特征不 同,金属元素含量也不同 ${ }^{[17,26]}$. 因此,在分析金属元素含

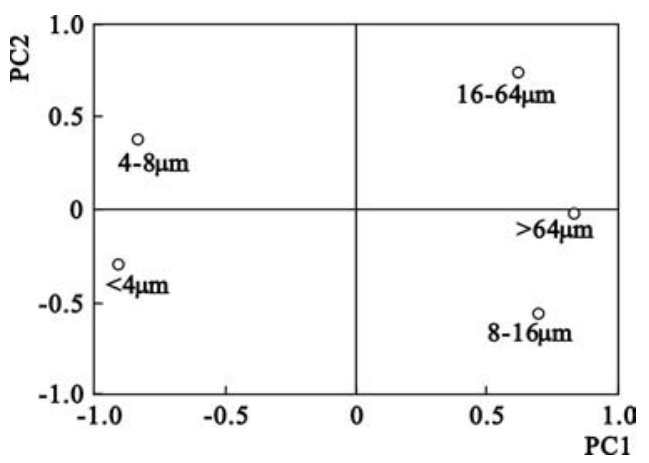

图 3 沉积物粒度主成分分析得分图

Fig. 3 Principal Component score plot of grain size 量变化时,应剔除由沉积物性质上的差异所造成的金属元素含量的变化,进行金属元素含量校正. 直接分析 某一粒级金属元素含量的方法在一定程度上破坏了金属元素原有的特征,而且操作过程比较繁琐; 本文选 用分析整个样品中金属元素含量, 然后校正到某一粒级金属元素含量的方法,探讨金属元素含量与沉积物 粒度变化的关系.

对沉积物金属元素含量进行校正通常是选取细颗粒含量作为标准;受水体沉积物性质的影响,对金属 元素含量进行校正所选取的粒级标准归纳起来有 $<2 \mu \mathrm{m} 、<4 \mu \mathrm{m} 、<16 \mu \mathrm{m} 、<20 \mu \mathrm{m}$ 、 $<63 \mu \mathrm{m}$ 等, 这主要是根 据沉积物粒度组成与金属元素含量的相关性进行选取 ${ }^{[2-4,13,17-22]}$. 太湖沉积物粒度组成以粉砂和粘土为 主, 对 MS 岩芯金属元素含量与粒度组分进行相关分析, 结果表明,第一类元素与粘土等细颗粒含量具有较 好的正相关性, $\mathrm{Na}$ 与粗颗粒 ( >16 $\mu \mathrm{m}$ ) 含量具有较好的正相关性, 与粘土含量呈显著负相关 (表 1). 根据元 素含量与不同粒级含量的相关性, 以及沉积物粒度主成分分析结果, 选取粘土组分含量进行金属元素含量 校正. 校正方法为:金属元素 $(\mathrm{Na}$ 除外) 校正含量 $=$ 样品中元素含量 $/$ 粘土百分含量, $\mathrm{Na}$ 的校正含量 $=$ 样品 中 $\mathrm{Na}$ 的含量/( 1 - 粘土百分含量).

除了采用沉积物粒度对金属元素含量进行校正之外, $\mathrm{Al} 、 \mathrm{Fe} 、 \mathrm{Cs} 、 \mathrm{Sc} 、 \mathrm{Co}$ 和 $\mathrm{Ti}$ 等元素也常作为沉积物元 素含量校正的参比元素 ${ }^{[3,4,17,20,22]}$. 如果沉积物中金属元素含量的变化是由沉积物粒度变化引起的,采用参 比元素对金属元素含量进行校正后的结果应该与采用粒度校正的结果基本一致 ${ }^{[16]}$. Al 在表生地质过程中 地球化学性质不活泼,其含量几乎完全受陆源细小的铝硅酸盐碎屑所控制, 在参考元素选取上应用较广 
泛 $^{[3,4,17,20,22]}$. 采用 $\mathrm{Al}$ 对金属元素含量进行校正方法为: 金属元素 ( $\mathrm{Na}$ 除外) 校正含量 = 样品中元素含量/ 样品中 $\mathrm{Al}$ 的含量, $\mathrm{Na}$ 的校正含量 $=$ 样品中 $\mathrm{Na}$ 的含量 $\times \mathrm{Al}$ 的含量 $/($ 沉积岩芯中 $\mathrm{Na}+\mathrm{Al}$ 的平均含量).

表 1 MS 岩芯金属元素及粒度相关分析 *

Tab. 1 The correlation coefficient of metals and grain size

\begin{tabular}{|c|c|c|c|c|c|c|c|c|c|c|c|c|c|c|c|c|c|c|c|c|c|}
\hline & $\mathrm{Al}$ & $\mathrm{Ba}$ & $\mathrm{Be}$ & $\mathrm{Ca}$ & $\mathrm{C}_{0}$ & $\mathrm{Cr}$ & $\mathrm{Cu}$ & $\mathrm{Fe}$ & $\mathrm{K}$ & $\mathrm{Mg}$ & Mn & $\mathrm{Na}$ & $\mathrm{Ni}$ & $\mathrm{Pb}$ & $\mathrm{Sr}$ & V & $\mathrm{Zn}$ & $<4 \mu \mathrm{m}$ & $48 \mu \mathrm{m}$ & $8-16 \mu \mathrm{m}$ & $16.64 \mathrm{um}$ \\
\hline $\mathrm{Ba}$ & 0.93 & & & & & & & & & & & & & & & & & & & & \\
\hline $\mathrm{Be}$ & 0.94 & 0.88 & & & & & & & & & & & & & & & & & & & \\
\hline $\mathrm{Ca}$ & 0.75 & 0.52 & 0.65 & & & & & & & & & & & & & & & & & & \\
\hline Co & 0.87 & 0.85 & 0.92 & 0.46 & & & & & & & & & & & & & & & & & \\
\hline $\mathrm{Cr}$ & 0.94 & 0.87 & 0.98 & 0.65 & 0.93 & & & & & & & & & & & & & & & & \\
\hline $\mathrm{Cu}$ & 0.12 & 0.31 & 0.21 & -0.45 & 0.36 & 0.18 & & & & & & & & & & & & & & & \\
\hline $\mathrm{Fe}$ & 0.94 & 0.86 & 0.96 & 0.68 & 0.91 & 0.98 & 0.14 & & & & & & & & & & & & & & \\
\hline $\mathrm{K}$ & 0.97 & 0.87 & 0.96 & 0.79 & 0.84 & 0.95 & 0.06 & 0.96 & & & & & & & & & & & & & \\
\hline $\mathrm{Mg}$ & 0.93 & 0.78 & 0.90 & 0.91 & 0.76 & 0.90 & -0.15 & 0.91 & 0.96 & & & & & & & & & & & & \\
\hline Mn & 0.96 & 0.85 & 0.94 & 0.82 & 0.83 & 0.94 & 0.01 & 0.96 & 0.98 & 0.97 & & & & & & & & & & & \\
\hline $\mathrm{Na}$ & -0.89 & -0.81 & -0.93 & -0.57 & -0.86 & -0.94 & -0.29 & -0.94 & -0.91 & -0.84 & -0.90 & & & & & & & & & & \\
\hline $\mathrm{Ni}$ & 0.94 & 0.84 & 0.96 & 0.75 & 0.89 & 0.97 & 0.09 & 0.96 & 0.95 & 0.94 & 0.97 & -0.92 & & & & & & & & & \\
\hline $\mathrm{Pb}$ & -0.11 & 0.09 & -0.02 & -0.48 & 0.04 & -0.03 & 0.62 & -0.09 & -0.17 & -0.28 & -0.13 & -0.07 & -0.03 & & & & & & & & \\
\hline $\mathrm{Sr}$ & 0.63 & 0.46 & 0.45 & 0.91 & 0.31 & 0.45 & -0.49 & 0.48 & 0.61 & 0.74 & 0.64 & -0.32 & 0.55 & -0.51 & & & & & & & \\
\hline $\mathrm{V}$ & 0.93 & 0.87 & 0.98 & 0.59 & 0.96 & 0.99 & 0.26 & 0.97 & 0.93 & 0.87 & 0.92 & -0.94 & 0.96 & -0.01 & 0.39 & & & & & & \\
\hline $\mathrm{Zn}$ & 0.89 & 0.82 & 0.90 & 0.72 & 0.78 & 0.91 & 0.09 & 0.90 & 0.90 & 0.90 & $0.94-$ & 0.89 & 0.96 & 0.13 & 0.52 & 0.88 & & & & & \\
\hline$<4 \mu \mathrm{m}$ & 0.77 & 0.69 & 0.80 & 0.54 & 0.78 & 0.81 & 0.24 & 0.80 & 0.79 & 0.74 & $0.77-$ & 0.83 & 0.82 & 0.01 & 0.34 & 0.82 & 0.75 & & & & \\
\hline $4-8 \mu \mathrm{m}$ & 0.63 & 0.64 & 0.68 & 0.27 & 0.69 & 0.69 & 0.41 & 0.64 & 0.62 & 0.53 & $0.58-$ & 0.71 & 0.65 & 0.22 & 0.11 & 0.70 & 0.62 & 0.85 & & & \\
\hline $8-16 \mu \mathrm{m}$ & -0.50 & -0.29 & -0.46 & -0.80 & -0.35 & -0.50 & 0.47 & -0.54 & -0.59 & -0.71 & -0.61 & 0.40 & -0.57 & 0.54 & -0.69 & -0.44 & -0.50 & -0.50 & -0.12 & & \\
\hline $16-64 \mu \mathrm{m}$ & -0.43 & -0.51 & -0.50 & 0.04 & -0.58 & -0.50 & -0.60 & -0.45 & -0.39 & -0.25 & -0.35 & 0.57 & -0.44 & -0.40 & 0.17 & -0.54 & -0.41 & -0.73 & -0.92 & -0.18 & \\
\hline$>64 \mu \mathrm{m}$ & -0.738 & -0.70 & -0.73 & -0.66 & -0.60 & -0.71 & -0.15 & -0.68 & -0.75 & -0.74 & -0.75 & 0.71 & -0.75 & -0.01 & -0.56 & -0.70 & -0.75 & -0.72 & -0.65 & 0.34 & 0.01 \\
\hline
\end{tabular}

* 相关系数大于 0.25 时, 在 0.01 水平显著相关; 相关系数大于 0.20 时,在 0.05 水平显著相关.

MS 岩芯中金属元素含量经沉积物粒度(粘土)、Al 元素含量校正后,整个沉积岩芯中元素含量变化已 较为稳定 (图 4). 由于沉积物中金属元素与细颗粒结合过程是物理化学生物过程的综合作用 ${ }^{[20]}$, 沉积物性 质 (粒度、矿物等) 对金属元素含量变化的影响系数为 $80 \%-90 \%{ }^{[27]}$; 同时矿物分析也表明,沉积岩芯 $0-$ $14 \mathrm{~cm}$ 与 $14-50 \mathrm{~cm}$ 相比, 石英、钠长石含量明显增加 ${ }^{[24]}, \mathrm{Na} / \mathrm{K}$ 比值升高, CIA 降低, 反映了沉积物风化程 度的降低 ${ }^{[28]}$. 因此,沉积岩芯 $0-14 \mathrm{~cm}$ 金属元素含量的变化是与沉积物风化程度降低导致的粒度变粗、所 吸附的金属元素含量降低、以及石英 $\left(\mathrm{SiO}_{2}\right)$ 等矿物含量的增加对金属元素产生稀释作用有关 ${ }^{[19,20]}$. 由于沉 积物金属元素之间、以及与沉积物粒度之间变化关系的复杂性, MS 岩芯金属元素含量经沉积物粒度、Al 元 素校正后,岩芯上部某些元素含量增大/减小的趋势未完全改变; 对比采用沉积物粒度、 $\mathrm{Al}$ 元素校正的结 果, 采用沉积物粒度校正后 MS 岩芯金属元素含量的变化更为稳定,应采用粘土含量对金属元素含量进行 校正 $^{[16]}$.

MS 岩芯金属元素含量经沉积物粒度、Al 元素校正后, 岩芯表层 $4 \mathrm{~cm}$ 中 $\mathrm{Pb} 、 \mathrm{Zn} 、 \mathrm{Ni}$ 等元素含量仍较高, 说明表层沉积物中 $\mathrm{Pb} 、 \mathrm{Zn} 、 \mathrm{Ni}$ 等元素含量除了受沉积物粒度组成的影响之外,还受其他因素的影响; MS 岩 芯中有机质含量较稳定, 对金属元素含量影响较弱 ${ }^{[15]}$. 因此, 表层沉积物中 $\mathrm{Pb} 、 \mathrm{Zn} 、 \mathrm{Ni}$ 等元素含量较高与人 为污染有关 ${ }^{[7]}$. 经沉积物粒度、 $\mathrm{Al}$ 元素校正后,金属元素含量的变化可以客观的反映金属元素的人为污染 特征 ${ }^{[4]}$. 在进行沉积物金属元素人为污染研究时, 金属元素含量采用沉积物粒度、 $\mathrm{Al}$ 元素校正后, 把人为活 动强度相对较弱的时期作为背景值, 可以判断人为活动的贡献强度. 


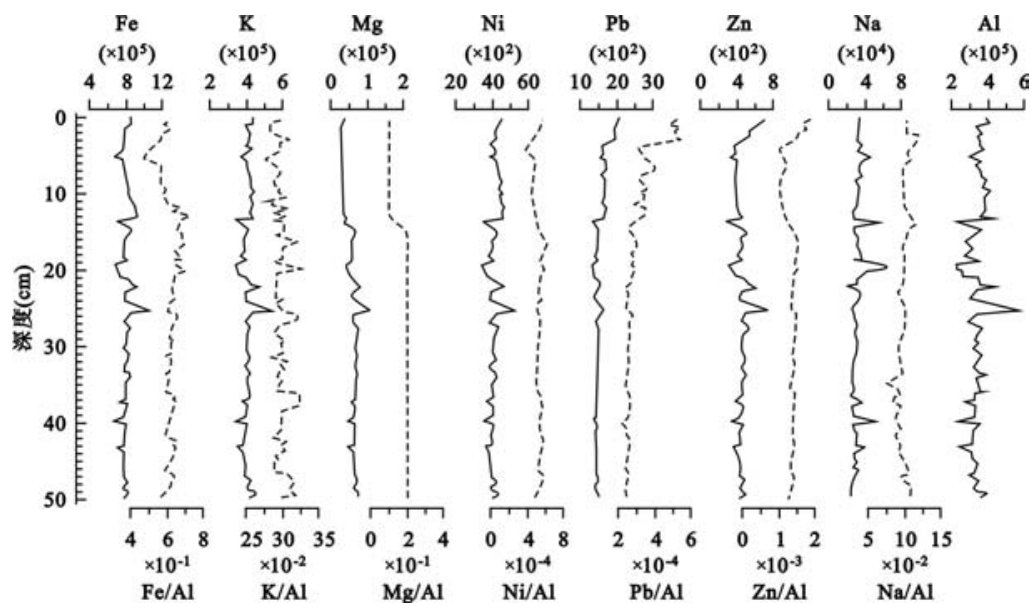

图 4 采用粘土含量 (实线)、 $\mathrm{Al}$ 元素含量(虚线)标准化后 MS 岩芯金属元素变化特征

Fig. 4 The variation of metals contents normalized by the content of clay and element $\mathrm{Al}$ in Core MS

\section{3 结论}

太湖 $\mathrm{MS}$ 岩芯中 $\mathrm{Al} 、 \mathrm{~K} 、 \mathrm{Fe}$ 等元素含量在岩芯中下部含量较高, 岩芯上部含量较低, $\mathrm{Na}$ 含量变化规律与 $\mathrm{Al} 、 \mathrm{~K} 、 \mathrm{Fe}$ 等相反. $\mathrm{MS}$ 沉积岩芯金属元素含量的变化主要与化学风化作用减弱导致的沉积物粒度组成变粗 有关,粘土含量是影响金属元素含量变化的主要组分. 经粘土含量校正后, MS 岩芯金属元素具有一致的变 化规律,并可以客观的反映表层沉积物重金属元素的人为污染特征. 在对太湖沉积物金属元素含量进行对 比分析、污染评价时,应该消除沉积物粒度组成对金属元素含量的影响; 就 MS 岩芯而言,采用沉积物粒度 (粘土含量)对金属元素含量进行校正后的结果要比采用参比元素校正的结果更为理想.

\section{4 参考文献}

[1] Allen H E, Fu G, Deng B. Analysis of acid-volatile sulfide (AVS) and simultaneously extracted metals (SEM) for the estimation of potential toxicity in aquatic sediments. Environ Toxicol \& Chem, 1993, 12 : $1441-1453$.

[2] Howari F M, Banat K M. Assessment of Fe, Zn, Cd, Hg and Pb in the Jordan and Yarmouk River sediment in relation to their physicochemical properties and sequential extraction characterization. Water Air \& Soil Pollut, 2001, 132: $43-59$.

[3] Green-Ruiz C, P ez-Osuna F. Heavy metal anomalies in lagoon sediments related to intensive agriculture in Altata-Ensenada del Pabell n coastal system (SE Gulf of California). Environ Int, 2001, 26 : 265 - 273.

[4] Roussiez V, Ludwig W, Probst J L, et al. Background levels of heavy metals in surficial sediments of the Gulf of Lions ( NW Mediterranean) : an approach based on ${ }^{133} \mathrm{Cs}$ normalization and lead isotope measurements. Environ Pollut, 2005, 138: 167 - 177.

[5] Rose N L, Boyle J F, Du Y, et al. Sedimentary evidence for changes in the pollution status of Taihu in the Jiangsu region of eastern China. J Paleolimnol, 2004, 32: 41 - 51.

[6] 袁旭音, 陈 骏, 季峻峰等. 太湖沉积物和湖岸土壤的污染元素特征及环境变化效应. 沉积学报, $2002, \mathbf{2 0}(3)$ : $427-434$.

[7] 刘恩峰, 沈 吉, 刘兴起等. 太湖 MS 岩芯重金属元素地球化学形态研究. 地球化学, 2004, 33(6): $602-610$.

[8] 朱广伟, 秦伯强, 高 光等. 太湖近代沉积物中重金属元素的累积. 湖泊科学, 2005, 17(2): 143 -150 . 
[9] Qu W C, Dickman M, Wang S M. Multivariate analysis of heavy metal and nutrient concentrations in sediments of Lake Taihu, China. Hydrobiologia, 2001, 450: 83 - 89.

[10] Wang X C, Yan W D, An Z, et al. Status of trace elements in paddy soil and sediment in Lake Taihu region. Chemosphere, 2003, 50: $707-710$.

[11] 王 海, 王春霞, 王子健. 太湖表层沉积物中重金属的形态分析. 环境化学, 2002, 21 (5): 430 -435 .

[12] Yang H D, Rose N L, Battarbee R W. Distribution of some trace metals in Lochnagar, a Scottish mountain lake ecosystem and its catchment. Sci Total Environ, 2002, 285: 197 - 208.

[13] 陈静生, 陶 澍, 邓宝山等. 水环境化学. 北京: 高等教育出版社, 1987: $181-188$.

[14] Guerra-García J M, García-Gómez J C. Assessing pollution levels in sediments of a harbour with two opposing entrances: Environmental implications. J Environ Manage, 2005, 77: 1 - 11.

[15] Nguyen H L, Leermakers M, Os n J, et al. Heavy metals in Lake Balaton: water column, suspended matter, sediment and biota. Sci Total Environ, 2005, 340: 213230.

[16] Suh J Y, Birch G F. Use of grain-size and elemental normalization in the interpretation of trace metal concentrations in soils of the reclaimed area adjoining Port Jackson, Sydney, Australia. Water Air \& Soil Pollut, 2005, 160: 357371.

[17] Huang W W, Zhang J. Effect of particle size on transition metal concentrations in the Changjiang (Yangtze River) and Huanghe (Yellow River), China. Sci Total Environ, 1990, 94: 187 - 207.

[18] Soto-Jim nez M F, P ez-Osuna F. Distribution and Normalization of Heavy Metal Concentrations in Mangrove and Lagoonal Sediments from Mazatl n Harbor ( SE Gulf of California). Estuar Coast \& Shelf Sci, 2001, 53: $259-274$.

[19] Zhang C S, Wang L J, Li G S, et al. Grain size effect on multi-element concentrations in sediments from the intertidal flats of Bohai Bay, China. Appl Geochem, 2002, 17: 59 - 68.

[20] Chen Z Y, Saito Y, Kanai Y, et al. Low concentration of heavy metals in the Yangtze estuarine sediments, China: a diluting setting. Estuar Coast \& Shelf Sci, 2004, 60: 91 - 100.

[21] 陈静生. 论小于 $63 \mu \mathrm{m}$ 粒级作为水体颗粒物重金属研究介质的合理性及有关粒级转换模型研究. 环 境科学学报, 1994, 14(4): 419-425.

[22] 刘素美, 张 经. 沉积物中重金属的归一化问题一以 $\mathrm{Al}$ 为例. 东海海洋, 1998, 16(3): $48-55$.

[23] 孙顺才, 黄渏平. 太湖. 北京: 海洋出版社, 1993: $21-224$.

[24] 刘恩峰. 长江中下游典型湖泊沉积物地球化学特征及人类活动响应一以太湖、太白湖为例. 中国科 学院南京地理与湖泊研究所博士学位论文, 2005.

[25] 徐 馨, 何才华, 沈志达等. 第四纪环境研究方法. 贵阳: 贵州科技出版社, 2001: 74 .

[26] Wong G T F, Moy C S. Cesium-137, metals and organic carbon in the sediments of James River Estuary, Virginia. Estuar Coast \& Shelf Sci, 1984, 18: 37 - 49.

[27] Zhang J. Heavy metal compositions of suspended sediments in the Changijiang( Yangtze River) estuary: Significance of riverine transport to the ocean. Cont Shelf Res, 1999, 19: $1521-1543$.

[28] Nesbitt H W, Young G M. Early Proterozoic climates and plate motions inferred from major element chemistry of lutites. Nature, 1982, 299: $715-717$. 